\title{
Are Heimlich maneuver videos on YouTube accurate and reliable?
}

BY ETHEM ACAR, Ö.DOĞAN ALATAS, AHMET DEMIR, ULVIYE KIRLI, CEM YALIN KILINC, ISMAIL KIRLI, HALIL BEYDILLI, BIRDAL YILDIRIM

\section{Abstract}

Introduction. First aid for airway obstruction is a life-saving maneuver that can be implemented by anyone. In this study, we determined the accuracy of Heimlich maneuver videos posted on the Internet.

Materials and methods. Heimlich maneuver videos uploaded on to YouTube were evaluated. We recorded by whom the video was uploaded, upload time, the number of viewers, and to whom it was intended. Scores from o to 7 were used to evaluate video suitability. Data were analyzed using SPSS 20.0 for Windows software. A $p$-value $<0.05$ was considered to indicate significance.

Results. A total of 640 videos were evaluated; 466 (72.8\%) videos were excluded because their content was primarily for entertainment purposes. In total, 174 videos met the inclusion criteria and were subjected to analysis. Of the 174 videos analyzed, 54(31\%) were uploaded anonymously, the mean number of viewers was $26,814 \pm 4,860$, and the median video duration was $4.19 \mathrm{~min}$ (range, o.06-114 min). The mean video score was $2.7 \pm 1.6$. Using this value as a cut-off, a significant relationship between reliability and uploading institution was detected $(\mathrm{p}$ 
$\leq$ 0.05), but not between the number of views and reliability ( $p=0.428)$.

Conclusion. Our results suggest that Heimlich maneuver videos uploaded to YouTube were not particularly educational because only $13 \%$ of the videos received an above-average score.

Key words: airway obstruction, first aid, YouTube video

\section{Introduction}

Without rapid intervention, airway obstruction caused by complete blockage of the airway by a foreign object leads to death because the patient cannot breathe, bruising develops, and respiratory and cardiac arrest ensues. (1) Back blows, abdominal thrusts, and chest thrusts are recommended by the International Liaison Committee on Resuscitation (ILCOR) guidelines for a conscious patient with an obstructed airway, whereas abdominal and chest thrusts are recommended for unconscious patients. These maneuvers increase intrathoracic pressure and can prevent death by ejecting the foreign body from the mouth. $(2,3)$

Both healthcare and non-healthcare workers should be aware of these life-saving maneuvers, and organized courses are available on this subject. The Internet can also facilitate with learning, as Heimlich maneuver videos are available on many social networking sites and can be viewed by anyone.

YouTube, on which people upload and share videos, is one of the most popular social networking sites. YouTube videos are an important platform for sharing relevant healthcare information due to their wide accessibility, but the risk of disseminating misleading information should not be ignored. (4-6)

In this study, we watched 640 Heimlich maneuver videos uploaded on to YouTube. The objective of this study was to determine the accuracy and reliability of Heimlich maneuver videos posted on YouTube. 


\section{Materials and methods}

We evaluated Heimlich maneuver videos uploaded on to YouTube. YouTube was queried using the search terms "Heimlich maneuver", and the following exclusion criteria were applied: 1) medically related but not to the Heimlich maneuver; 2) no application mentioned; 3) not in English; 4) not educationally related or part of a news story; 5) included advertising content (courses advertised etc.); 6) entertaining or funny; 7) contained non-medical material; and 8) repeat video.

The data collected in this study included the source of the video; the video duration; number of viewers; inclusion of humans, manikins, or both; and whether the maneuver was described using photographs or diagrams. Videos were categorized by source into five groups of guidelines; i.e., the American Heart Association/Red Cross/ European Resuscitation Council, or ILCOR; professionals, such as physicians associated with the subject; individuals with unspecified credentials; agencies (news programs); and corporations (corporations advertising courses).

The videos were viewed and evaluated by two experienced emergency medicine specialists who were blinded to the other's review. The portion of the ILCOR Adult Basic Life Support course on foreign body obstruction was summarized and read by the researchers.

The guidelines specify the following for performing the Heimlich maneuver. Back blows, abdominal thrusts, and chest thrusts are recommended for conscious patients with an obstructed airway, whereas abdominal thrusts and chest thrusts are recommended for unconscious patients. In addition, the Heimlich maneuver has been described in detail for infants and children. (3) A scoring system of $0-7$ was applied to assess the suitability of the video (table 1). When the two experts scored the same video differently, it was evaluated by a third experienced emergency medicine faculty member to reach a decision on suitability. 


\section{Statistical analysis}

Data were analyzed using SPSS 20.0 for Windows software (SPSS Inc., Chicago, IL, USA). Normality of the quantitative data distribution was assessed using the Kolmogorov-Smirnov test. Parametric tests (independent-sample $t$-test and post hoc Tukey's test) were used for normally distributed data, and non-parametric tests (Mann-Whitney $U$ test and the Kruskal-Wallis test) were applied to data not normally distributed. Continuous data are presented as means \pm standard deviations or medians and ranges, as appropriate. All tests were twotailed, and a $p$-value $<0.05$ was considered to indicate significance.

\section{Results}

A total of 640 videos were identified using "Heimlich maneuver" as keywords on YouTube in February 2015. A total of 466 (72.8\%) videos were excluded, the majority because their content was entertaining in nature (table 2).

Of the 174 videos analyzed, 54 (31\%) were uploaded anonymously, and 51(29.3\%) were uploaded by official institutions. The total number of viewers was $26,814 \pm 4,860$, and the median elapsed time of the videos was $4.19 \mathrm{~min}$ (range, $0.06-114 \mathrm{~min}$ ). Thirty-six(20.6\%) videos were uploaded in 2013, one was uploaded in 2006, and 125(71.8\%) videos involved real people (table 3 ). The evaluation scores are provided in table 3 .

Only seven (4\%) videos scored the maximum of 7 points; five were uploaded by public institutions and one was uploaded by a health professional. The mean video score was $2.7 \pm 1.6$. Videos with a score $\geq 5$ were considered above average and reliable. When we used this value as a statistical cut-off, a significant relationship was detected between reliability and uploading institution $(Z:-2.451, p \leq 0.05)$ but not between the date of upload and reliability $(Z:-1.209, p=0.428)$. The time and number of monitoring of videos were significantly related to reliability 
( $Z:-2.775, Z:-2.086, p \leq 0.05$ respectively). When we divided the videos into two groups, one uploaded by public institutions-health professionals, the other by others, no difference was found between uploading institution and video display rate, date, time, or whether actual persons were used in the video ( $(t:-0.751, p=0.454),(t: 0.411, p$ : 569), (t:1.74, $p: 0.84)$, and (t:0.023, $p: 0.982)$ respectively).

\section{Discussion}

Our results suggest that Heimlich maneuver videos on YouTube are not useful for educational purposes, as only $13 \%$ received an above-average score.

YouTube is one of the most popular social networking sites for uploading and sharing of videos. Since its establishment in 2005, large amounts of useful information have been obtained by searching YouTube. However, the accuracy of the information can be suspect. Yaylaci et al. (4) investigated the reliability and accuracy of cardiopulmonary resuscitation (CPR) and basic life support (BLS) videos on YouTube. They found 1,994 uploaded videos on these two subjects and reported that 209 were appropriate based on the 2010 guidelines. They also stated that few videos were considered excellent in terms of educational value. Murugiah et al. (6) also searched for CPR and BLS videos on YouTube and reported no relationship between video accuracy and who uploaded the video or the number of views. They found that there was no relationship between the popularity of a video and its accuracy. Bezner et al. (7) searched for gastrochisis, congenital diaphragmatic hernia, pediatric inguinal hernia, and pectus excavatum videos on YouTube. They reported that most of the 40 videos identified were uploaded by healthcare professionals and that they were more accurate than those uploaded by others. Our results suggest that most of the Heimlich maneuver videos were not uploaded by healthcare professionals and that few were accurate and reliable. We found a significant relationship between reliability and accuracy and the person or institution that uploaded the video, which suggests that the videos that received the 
maximum score of 7 could be used by official institutions. The Heimlich maneuver is of interest to society as a whole. Thus, anyone interested in the subject can download and watch these videos. Therefore, videos shared by non-professionals may have low reliability and accuracy.

The 2010 guidelines on first aid for aspirating a foreign body state that the Heimlich maneuver is not more effective than hitting the back or compressing the chest and suggest that all three maneuvers should be used. However, abdominal thrusts can cause more complications than the other maneuvers. (3) However, of the maneuvers described in the videos for a patient who has aspirated a foreign object, the Heimlich maneuver videos scored the highest, followed by hitting the back in unconscious patients. First aid for an airway obstruction in infants and children was described in only $16 \%$ of the videos.

In conclusion, the Heimlich maneuver can save lives. Our results indicate that Heimlich maneuver videos uploaded by official institutions and professionals could facilitate the dissemination of accurate information about this important life-saving technique.

\section{References}

1. Tan H, Brown K, McGill T, Kenna MA, Lund DP, Healy GB. Airway foreign bodies (FB): a 10-year review. Int $\mathrm{J}$ Pediatr Otorhinolaryngol 2000;56(2):91-9.

2. Manno M. Pediatric respiratory emergencies: Upper airway obstruction and infections. In: Marx JA, Hockberger RS, Walls RM, et al, editors. Rosen's Emergency Medicine: Concepts and Clinical Practice. 7th ed. Philadelphia (PA): Elsevier Mosby. 2009. Chap. 166.

3. International Liaison Committee on Resuscitation. Part 2: Adult basic life support. Resuscitation 2005;67(2-3):187-201.

4. Yaylacı S, Serinken M, Eken C, Karcioglu O, Yilmaz A, Elicabuk H, et al. Are YouTube videos accurate and reliable on basic life support and cardio pulmonary resuscitation? Emerg Med Australas 2014;26(5):4747. 
5. Duncan I, Yarwood-Ross L, Haigh C. YouTube as a source of clinical skills education. Nurse Educ. Today 2013;33(12):1576-80.

6. Murugiah K, Vallakati A, Rajput K, Sood A, Challa NR. YouTube as a source of information on cardiopulmonary resuscitation. Resuscitation 2011;82(3):332-4.

7. Bezner SK, Hodgman EI, Diesen DL,Clayton JT, Minkes RK, Langer JC, et al. Pediatric Surgery on YouTubeTM: is the truth out there. $\mathrm{J}$ pediatr surg 2014;49(4):586-9.

Table 1. Scoring system applied to assess the suitability of the video.

TASK

Score

A1. Did he/she perform back blows in conscious patients?

A2. Did he/she perform abdominal thrusts in conscious patients?

A3. Was the patient placed supine?

A4: Did he/she perform abdominal thrusts in unconscious patients?

A5: Did he/she implement the Hemlich maneuver in children? 1

A6: Did he/she implement the Hemlich maneuver in infants? $\quad 1$

A7: Did he/she start CPR, when the patient didn't respond to the maneuver

CPR, cardiopulmonary resuscitation.

Table 2. The status of the video according to the exclusion criteria.

Reason of exclusion $\quad$ n $\%$

Medically related but not to the Heimlich maneuver

10015.6

No application mentioned

$54 \quad 8.4$

Not in the English language 
SIGNA VITAE 2016; 11(1):

Not educationally related or part of a news story $\quad 24 \quad 3.8$

Included advertising content (courses advertised

$18 \quad 2.8$ etc.

12

Entertaining or funny

30 .

O 2

Contained non-medical material

$50 \quad 7.8$

Repeat videos.

$54 \quad 8.4$

Not excluded

$17427 \cdot 3$

Total

640100

Table3. Characteristics of the videos included in the analysis.

\begin{tabular}{|l|ll}
\hline Date & n & \% \\
\hline 2006 & 1 & 0,6 \\
\hline 2007 & 6 & 3.4 \\
\hline 2008 & 18 & 10.3 \\
\hline 2009 & 14 & 8 \\
\hline 2010 & 15 & 8.6 \\
\hline 2011 & 26 & 14.9 \\
\hline 2012 & 20 & 11.5 \\
\hline 2013 & 36 & 20.7 \\
\hline 2014 & 26 & 14.9 \\
\hline 2015 & 12 & 6.9 \\
\hline Uploaded by & n & \% \\
\hline
\end{tabular}


SIGNA VITAE 2016; 11(1):

Official institutions (like AHA/ERC or University...) $\quad 51 \quad 29.3$

Healthcare professional(s) (physician, emergency medical technician, nurse etc.)

Individual with credentials unspecified

$54 \quad 31.5$

Private agency

74

News program

$18 \quad 410.3$

Performed on

n \%

Human

12571.8

Manikin

$34 \quad 19.5$

Both

$10 \quad 5 \cdot 7$

Described with pictures

$5 \quad 2.9$

Application

n $\%$

A1 correctly applied" Did he/she perform back blows in conscious patients?"

$70 \quad 40.2$

A2 correctly applied 'Did he/she perform abdominal thrusts in conscious patients?"

14985.6

A3 correctly applied 'Was the patient placed supine?"

$\begin{array}{ll}51 & 29.3\end{array}$

A4 correctly applied 'Did he/she perform abdominal thrusts in unconscious patients?"

$95 \quad 54.6$

A5 correctly applied 'Did he/she implement the Hemlich maneuver in children?"

2916.7

A6 correctly applied 'Did he/she implement the Hemlich maneuver in infants?"

A7 correctly applied 'Did he/she start CPR, when the patient didn't respond to the maneuver?" 
AHA, American heart association; CPR, cardiopulmonary resuscitation;

ERC, European resuscitation council.

Ethem Acar, Ö.Doğan Alatas, Halil Beydilli, Birdal Yıldırım

Departments of Emergency Medicine, Mugla Sitki Kocman University Medical Faculty, Mugla, Turkey

Ahmet Demir

Sultanbeyli State Hospital, Emergency Service, Istanbul, Turkey

Cem Yalin Kilinc

Department Of Orthopaedics-Traumatology, Mugla Sitki Kocman University Medical Faculty, Mugla, Turkey

İsmail Kırlı

Departments of Internal Medicine, Mugla Sitki Kocman University, Medical Faculty, Mugla, Turkey

Corresponding author:

Ethem Acar

Department of Emergency Medicine

Mugla Sitki Kocman University Medical Faculty

480oo, Kotekli, Mugla, Turkey

Phone: +9002522141323

Fax: +902522111345

E-mail: dr.ethemacar@hotmail.com

Article printed from Signa Vitae: http://www.signavitae.com

URL to article: http://www.signavitae.com/2016/o5/areheimlich-maneuver-videos-on-youtube-accurate-and-reliable/

Copyright (C) 2015 Signa Vitae. All rights reserved. 